\title{
A Novel Algorithm for Open-set Modulation Recognition of Communication Signals
}

\author{
Wang Bo, Huang Zhen \\ Space Center, Tsinghua University, Beijing, 100084, China \\ wangbo12@mails.tsinghua.edu.cn
}

Keywords: open-set modulation recognition, instant characteristic, high-order cumulants, classifier

\begin{abstract}
In this paper, a novel algorithm is proposed for open-set modulation recognition of communication signals by extracting the characteristics of received signals in the presence of additive white Gaussian noise. Some instant characteristics are defined, including continuous coefficient of unification instant phase, concentration ratio of instant amplitude unification and so on, meanwhile a two-stage classifier is also designed to improve recognition accuracy and avoid adding much computing time. The proposed recognition algorithm can identify nine different modulation modes from other unknown one, and noted that it is rarely sensitive to residual frequency offsets and available for MF signals. Simulation results demonstrate that the recognition accuracy of the proposed algorithm is better than $93 \%$ at $8 \mathrm{~dB}$ SNR value and robustness is also achieved for varied cosine roll-off signals.
\end{abstract}

\section{Introduction}

Automatic Modulation Recognition of communication signals is widely used in cognitive radio and context aware[1]. It is generally true that modulation recognition can be thought of as a pattern recognition problem on mathematics, using the characteristic parameters for pattern classification.

Overall, Modulation recognition can be divided into two main kinds, the one based on hypothesis testing and the another based on pattern recognition[2]. It is noted that hypothesis testing method has the simple decision rules and it is the optimal solution in theory[3]. However, a complex calculation and rich priori knowledge are required in the test of statistics. Although hypothesis testing method is suboptimal, it is easier to achieve and has better robustness[4]. In this paper, pattern recognition is used to extra characteristics.

The principle of pattern recognition method is to choose the various characteristics of received signals, set reasonable threshold value, and design classifier to judge different signals types. The characteristics include instantaneous frequency and amplitude, cumulants, transform domain features, fractal features and so on[5]. Classifier design is often achieves with decision tree classifier. On this aspect, the method of Azzouz and Nandi based on the characteristics of signal instantaneous method is most widely used, which proposes seven characteristics to recognize five modulation modes including AM, FM, FSK, PSK and ASK[6]. Azzouz`s method reaches the recognition accuracy exceed $90 \%$ in $15 \mathrm{~dB}$ SNR value[7] and other improved methods are also propose and better performances obtained.[8-9] However, the research methods above are directed at the baseband signal modulation mode recognition[10], which are more sensitive to residual frequency offsets. In this paper, a novel modulation recognition algorithms is proposed for classifying of the open-set communication signals, which is rarely sensitive to residual frequency offsets and available for MF signals. Meanwhile the cumulants are improved to reach a better robustness of square-root raised cosine roll-off digital filter

\section{Modulation Signal Recognition}


Algorithms Structures. This paper proposes a novel modulation recognition algorithms for classifying of the open-set communication signals, including pre-processing section and a two-stage modulation recognition section.

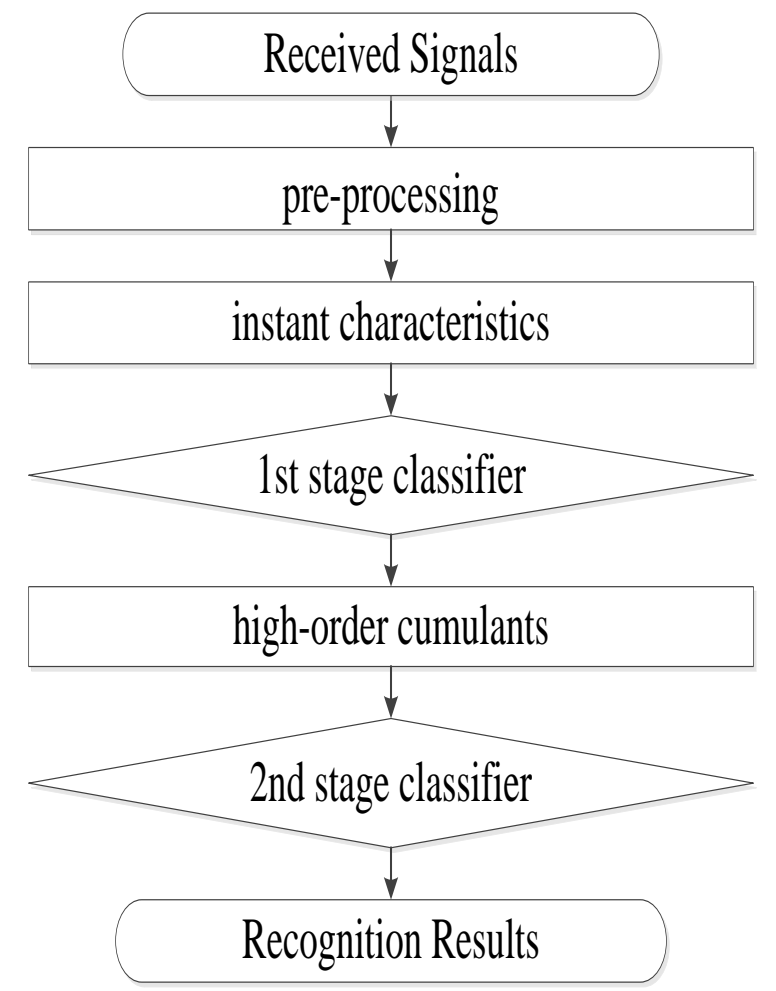

Fig.1: Algorithms structures including pre-processing section and a two-stage modulation recognition section

To be specific, power unification of received signals and instant signal parameter extraction are implemented in the pre-processing section. In the two-stage modulation recognition section, recognition of modulation modes is disposed in two steps. The instant characteristics are calculated and input into the first stage classifier. Next, if necessary, high-order cumulants are calculated and input into the second stage classifier to recognize some phase-modulated signals such as MPSK and QAM. Through the setting of the two-stage modulation recognition section, the amount of calculation is reduced and the algorithm is suitable for engineering application.

Pre-process. Pre-processing section includes power unification and instant signal parameter extraction, with two purposes as follows.

First, unifying input conditions of modulation recognition algorithm, and eliminating interference to algorithm caused by signal individual differences, to facilitate the extraction of main features of signals to be recognized, and improve the stability of the algorithm;

Second, computing common module extracted from different modulation signal features, reducing duplicate algorithm in algorithm operation, and reducing algorithm computation.

The distance and power of the signal transmitter terminal are different; accordingly, the power of input signal in the receiving terminal is different. In this way, power unification is necessary for subsequent recognition algorithms to extract signal amplitude information as required characteristic value. Through adding power unification factors in signals, the same average power can be achieved by different modulation modes (mapping modes).

After received by the antenna, RF signals are down-converted to intermediate frequency. Two signals of I-Q are formed, which are expressed as

$$
\begin{aligned}
& I_{n}=A(n T) g(n T) \cos \left(2 \pi \Delta f n T+\theta_{n}+\varphi_{0}\right) \\
& Q_{n}=A(n T) g(n T) \sin \left(2 \pi \Delta f n T+\theta_{n}+\varphi_{0}\right)
\end{aligned}
$$


Wherein, $A(n T)$ refers to amplitude modulation function, $g(n T)$ refers to transmitting terminal symbol filtering function, $\Delta f$ refers to intermediate frequency or residual frequency deviation, $\theta_{n}$ refers to phase modulation function, and $\varphi_{0}$ refers to initial phase.

Based on I-Q signals, signal instant amplitude and instant phase are extracted.

Instant amplitude is expressed below.

$a(n T)=\sqrt{I_{n}^{2}+Q_{n}^{2}}=A(n T) g(n T)$

Instant phase is expressed as

$\varphi(n T)=\arctan \left(\frac{I_{n}}{Q_{n}}\right)$.

\section{Calculating of Characteristic}

Based on the model analysis of the modulation signal, instant phase, and envelope features, with the principle of "simple calculation and outstanding features", the following seven characteristic values are used for modulation modes including AM, FM, ASK, FSK, MSK, BPSK, QPSK, OQPSK, and 16QAM.

Instant Characteristics. The first characteristic value $\gamma_{\max }$ is the maximum instant amplitude value in unification center, which is expressed as

$$
\gamma_{\max }=20 \lg \left[\frac{\max \left|F F T\left(a_{c n}(i)\right)\right|^{2}}{N}\right](d B)
$$

Wherein, $N$ refers to the number of samples of each signal segment, $a_{c n}(i)$ refers to instant amplitude in unification center, which is defined below.

$a_{c n}(i)=a_{n}(i)-1$

Wherein,

$a_{n(i)}=E\{a(i)\}$

$\gamma_{\max }$ is used to distinguish among signals that contain amplitude information (AM, ASK, BPSK, QPSK, 16QAM, etc.) or do not (FM, FSK, MSK). Modulation modes, including FM, FSK, MSK, contain no amplitude information. Their envelopes are approximately constant envelopes. Therefore, when $a_{c n}(i)$ is approximately equal to zero, the signal is determined to contain amplitude information. Besides, through the symbol filtering at the transmitting terminal, the envelopes of BPSK and QPSK also include amplitude information.

The second characteristic value $\sigma_{p}$ is continuous coefficient of unification instant phase. It is defined as

$$
\sigma_{p}=\sqrt{\frac{1}{N} \sum_{i=1}^{N}\left(\phi(i)-\frac{1}{N} \sum_{i=1}^{N} \phi(i)\right)^{2}}
$$

Wherein, $\phi(i)$ refers to the instant phase after smoothing filtering, which is defined as

$$
\begin{aligned}
& \phi(i+t)=K_{0}+K_{1} t(i)+\cdots+K_{m} t^{m}(i) \\
& t=\frac{\varphi-\varphi(i)}{\varphi(i+1)-\varphi(i)}
\end{aligned}
$$

Wherein, $\varphi(i)$ refers to instant phase. $\phi(i)$ parameter $\left\{K_{j}\right\}$ is determined in accordance with the law of least square method, namely:

The minimum value of

$$
\lambda\left(K_{0}, K_{1}, \ldots, K_{m}\right)=\sum_{t=-n}^{n}\left(\phi(i+t)-\sum_{j=0}^{m} K_{j} t^{j}\right)^{2}
$$

is taken as $\frac{\partial \lambda}{\partial K_{k}}=0,(k=0,1, \ldots, m)$.

$\sigma_{p}$ measures the continuous phase of instant phase distribution, and is used to characterize the continuously degree of instant phase distribution. $\sigma_{p}$ is used to distinguish between continuous phase variation modulation sub-ensemble (AM, ASK, MSK) and phase hopping modulation sub-ensemble on alternating code elements (BPSK, QPSK, 16QAM, FSK). 
The third characteristic value $\sigma_{f}$ is concentration ratio of instant amplitude unification. It is defined as follows:

$$
\sigma_{f}=\sqrt{\frac{1}{N} \sum_{i=1}^{N}\left(\chi(i)-\frac{1}{N} \sum_{i=1}^{N} \chi(i)\right)^{2}}
$$

Wherein, $\chi(i)$ refers to instant phase of the smoothed derivation after smoothing filtering, i.e. the derivative of $\phi(i)$, which is expressed as

$$
\chi(i)=\frac{d \phi(t)}{d t}
$$

$\sigma_{f}$ measures the intensity phase of instant phase distribution, and is used to characterize the intensity degree of instantaneous frequency. $\sigma_{f}$ is used to distinguish between the analog frequency modulation signal (FM) and digital frequency modulation signal (FSK, MSK).

The fourth characteristic value $\sigma_{a}$ is concentration ratio of signal envelope, which is using the following express

$$
\sigma_{a}=\frac{\frac{1}{N} \sum_{i=1}^{n}\left(A_{i}\right)^{4}}{\left(\frac{1}{N} \sum_{i=1}^{n}\left(A_{i}\right)^{2}\right)^{2}}
$$

$\mu_{a}$ is used to distinguish between the analog amplitude-modulated signal (AM) and digital amplitude-modulated signal (ASK). $\mu_{a}$ measures the intensity phase of instant amplitude distribution. Therefore, the characteristic value is used to distinguish signals like AM whose instant amplitude is continuously distributed, and signals like ASK whose instant amplitude is distributed in several discrete values.

High-order Cumulants. The fifth to seventh characteristic values are acquired based on four orders of signals to be recognized, which are expressed as $\left|C_{4,0}\right|,\left|C_{4,1}\right|$, and $\left|C_{4,2}\right|$.

The signal modulation modes of the digital phase-modulated signal and the QAM signal are more complex than those of other signals. QAM signals, especially, when recognizing 16QAM, the extracted characteristic value is substantially the same as QAM modulation mode of other order. Therefore, this type of signals is subdivided by taking higher-order cumulants as signal characteristic at the second order. The traditional high-order cumulants method are analyzed and improve since there's no need of priori information of signal carrier frequency.

To be specific, for stable random process of certain composite zero average $X(k)$, the four-order cumulants are expressed as

$$
\begin{aligned}
& C_{4,0}=M_{4,0}-3 M_{2,0}^{2} \\
& C_{4,1}=M_{4,1}-3 M_{2,1} M_{2,0} \\
& C_{4,2}=M_{4,2}-\left|M_{2,0}\right|^{2}-2 M_{2,1}^{2} .
\end{aligned}
$$

Wherein, $M_{p, q}=E\left[X(k)^{p-q}\left(X(k)^{*}\right)^{q}\right]$.

In this paper, intermediate frequency signal or residual frequency deviation signal are analyzed, and the cumulants of MPSK and QAM with intermediate frequency are acquired. The mode is showed in the following table as characteristic value.

Table 1: High-order Characteristic Value of BPSK, QPSK, 16QAM with Residual Frequency Offsets

\begin{tabular}{cccc} 
& $\left|C_{4,0}\right|$ & $\left|C_{4,1}\right|$ & $\left|C_{4,2}\right|$ \\
\hline BPSK & 0 & 0 & $E\lceil A(\cdot) g(\cdot)]^{4}$ \\
QPSK & 0 & 0 & $\frac{1}{4} E[A(\cdot) g(\cdot)]^{4}$ \\
16QAM & $E[A(\cdot) g(\cdot)]^{4}$ & $\frac{1}{3} E[A(\cdot) g(\cdot)]^{4}$ & $\frac{4}{3} E[A(\cdot) g(\cdot)]^{4}$ \\
A() means magnitude and $\mathrm{g}()$ means envelope of signals &
\end{tabular}




\section{Design of Classifier}

Based on the characteristics of each characteristic value and signal type, the two-stage tree classifier is designed as in Figure 2 and 3. Decision tree classifier is used.

Step 1: As is shown in Figure 2, based on the characteristic value $\gamma_{\max }$, first level classifier divides the signal into three categories: sub-category one (FM, FSK, MSK), sub-category two (OQPSK), and sub-category three (AM, ASK, BPSK, QPSK, 16QAM).

Step 2: $\sigma_{f}$ is used on sub-category one to separate frequency well-distributed FM. Since MSK is continuous phase modulation, $\sigma_{p}$ can be used to separate FSK and MSK.

Step 3: For sub-category three, AM and ASK signals are separated by characteristic value. AM and ASK are distinguished by $\sigma_{p}$. The recognition of MPSK and MQAM is implemented through secondary classification by characteristic values of higher-order cumulants.

Step 4: As is shown in Figure 3, BPSK, QPSK, and 16QAM is distinguished by high-order cumulants in the following way: using $\left|C_{4,2}\right|$ and $\left|C_{4,0}\right|$ to distinguish between QPSK and unknown signals, using $\left|C_{4,2}\right|$ and $\left|C_{4,1}\right|$ to recognize BPSK signals, and using $\left|C_{4,0}\right|$ and $\left|C_{4,2}\right|$ to separate 16QAM from unknown signals.

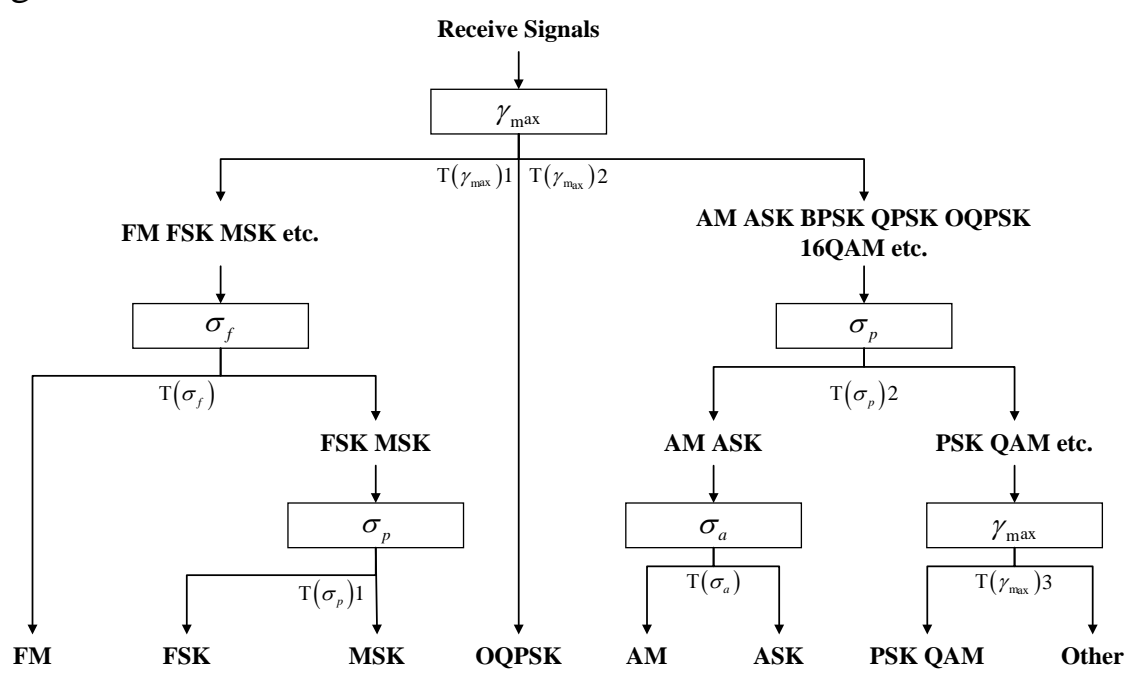

Fig.2: First Level Classifier of the Open-set Modulation Recognition $\mathrm{T}()$ means therefore of characteristics

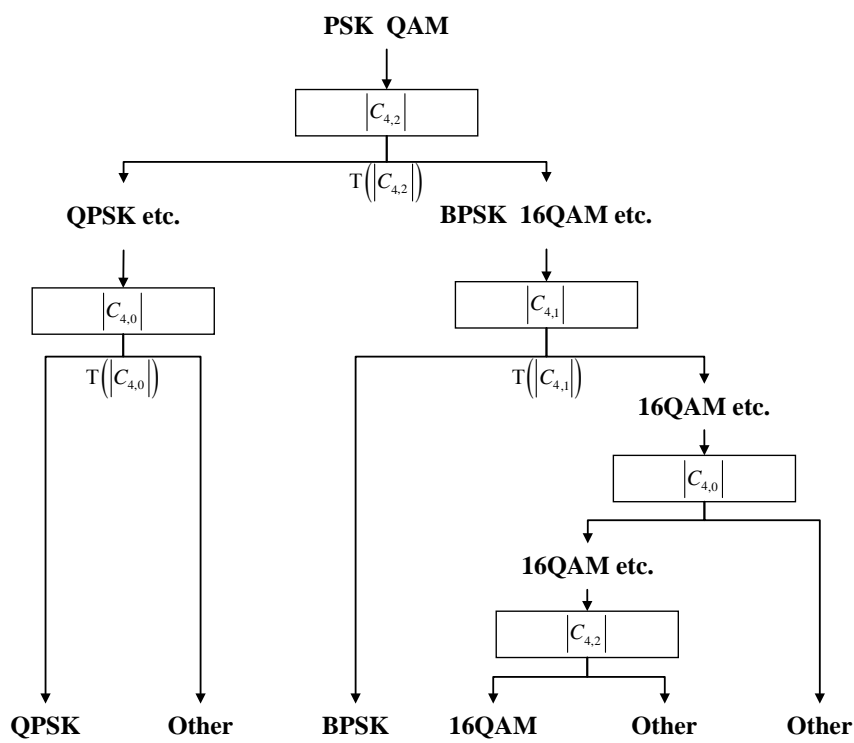

Fig.3: Second Level Classifier of the Open-set Modulation Recognition $\mathrm{T}()$ means therefore of characteristics 
Meanwhile, the best decision threshold value of each characteristic is determined through the simulation experiments, therefore, the distribution of characteristics under different SNRs is calculated, as is shown in Figure 4. The modulation modes of signals include AM, FM, ASK, FSK, MSK, BPSK, QPSK, OQPSK, 16QAM, and other common signals with the bandwidth of $1 \mathrm{MHz}$ and 1000 times per modulation mode. The results of therefore values are listed in Table 2.
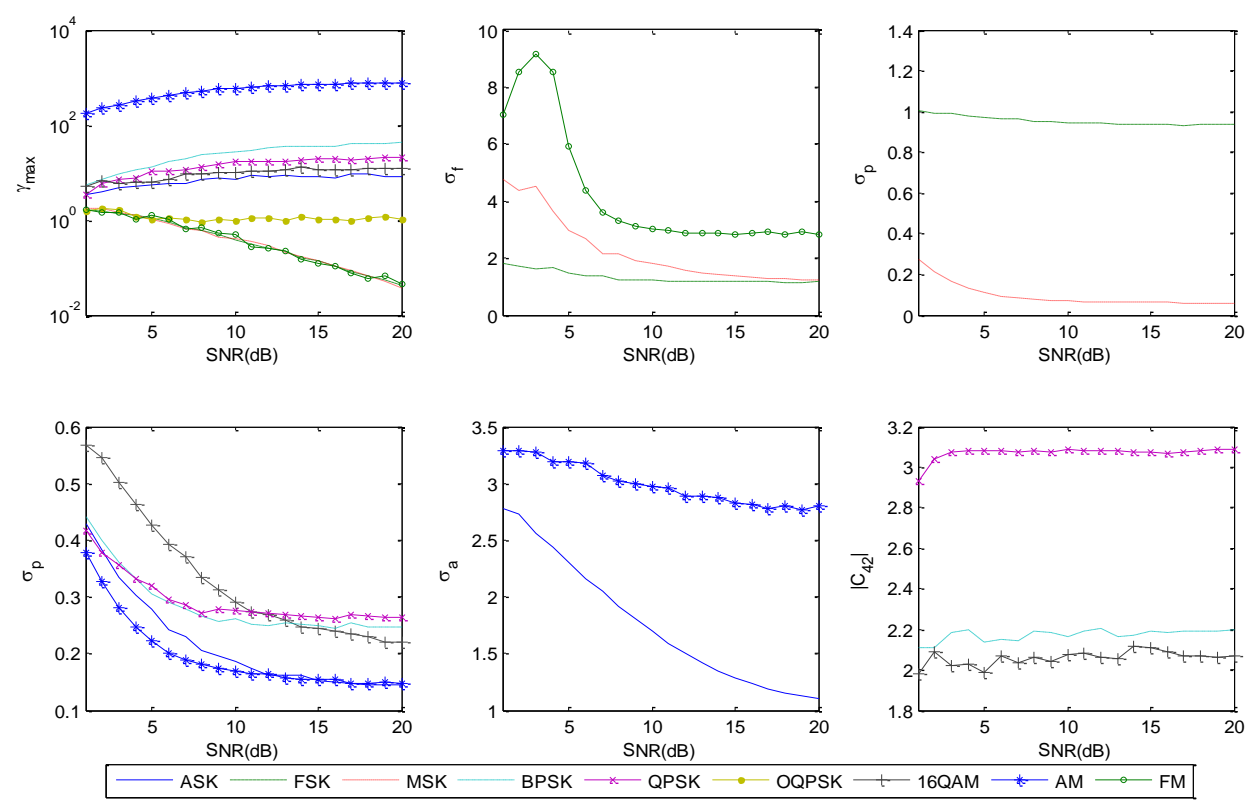

Fig.4: Distribution of Characteristics under Different SNRs

Signals include AM, FM, ASK, FSK, MSK, BPSK, QPSK, OQPSK, 16QAM and other common signals. SNR range is between $0 \mathrm{~dB}$ and $20 \mathrm{~dB}$

Table 2: Therefore Values of Characteristics

\begin{tabular}{cccc}
\hline Threshold & Value & Threshold & Value \\
\hline $\mathrm{T}\left(\gamma_{\max }\right) 1$ & 0.85 & $\mathrm{~T}\left(\sigma_{a}\right)$ & 2.25 \\
$\mathrm{~T}\left(\gamma_{\max }\right) 2$ & 3.00 & $\mathrm{~T}\left(\left|C_{4,2}\right|\right)$ & 2.60 \\
$\mathrm{~T}\left(\sigma_{f}\right)$ & 2.81 & $\mathrm{~T}\left(\left|C_{4,0}\right|\right)$ & 16.00 \\
$\mathrm{~T}\left(\sigma_{p}\right) 1$ & 0.50 & $\mathrm{~T}\left(\left|C_{4,1}\right|\right)$ & 1.95 \\
$\mathrm{~T}\left(\sigma_{p}\right) 2$ & 0.18 & $\mathrm{~T}\left(\gamma_{\max }\right) 3$ & 32.00 \\
\hline
\end{tabular}

\section{Simulation Analysis}

In this paper, the simulation analysis of open-set modulation recognition method in $8 \mathrm{~dB}$ SNR is carried out. First, nine kinds of modulation modes signals and other common ones with the bandwidth of $1 \mathrm{MHz}$ and residual frequency offset of $0.5 \mathrm{MHz}$ are generated. Particularly, the BPSK, QPSK and 16QAM signals have varied cosine roll-off coefficient ranging from 0.2 to 0.8 . Then, the adding noise signals are inputted into the algorithms to identify the modulation mode of signals, and statistic results are assessed finally.

Computer simulation results demonstrate that the proposed recognition algorithms can identify more species and reach much better recognition accuracy than Azzouz`s method in the same SNR value, residual frequency offset and cosine roll-off coefficient. Especially, it is noted that the algorithms identify nine different modulation modes and others; meanwhile, it reach the recognition accuracy better than $93 \%$ in $8 \mathrm{~dB}$ SNR value. The results show that significant performances the 
algorithms for signals with residual frequency offset and varied cosine roll-off coefficient in low SNR ratio can be obtained .

Table 3: Simulation Results $(\mathrm{SNR}=8 \mathrm{~dB}$, cosine roll-off coefficient $=0.2$ )

\begin{tabular}{ccccccccccc}
\hline & ASK & FSK & MSK & BPSK & QPSK & OQPSK & 16 QAM & AM & FM & Other \\
\hline (a) Method in this paper & $100 \%$ & $100 \%$ & $100 \%$ & $99 \%$ & $98 \%$ & $95 \%$ & $93 \%$ & $99 \%$ & $99 \%$ & $96 \%$ \\
(b) Azzouz's Method & $93 \%$ & $81 \%$ & - & $88 \%$ & $82 \%$ & - & - & $85 \%$ & $81 \%$ & - \\
(c) Method in this paper & $100 \%$ & $100 \%$ & $100 \%$ & $99 \%$ & $98 \%$ & $95 \%$ & $94 \%$ & $99 \%$ & $100 \%$ & $96 \%$ \\
(d) Azzouz's Method & $98 \%$ & $99 \%$ & - & $93 \%$ & $91 \%$ & - & - & $87 \%$ & $85 \%$ & - \\
\hline
\end{tabular}

Signals of $1 \mathrm{MHz}$ bandwidth under $8 \mathrm{~dB}$ SNR, 1000 times per modulation mode. (a)(b) with $0.5 \mathrm{MHz}$ residual frequency offset and (c)(d) without that. The cosine roll-off coefficient of BPSK, QPSK and 16QAM is 0.2 .

Table 4: Simulation Results $(\mathrm{SNR}=8 \mathrm{~dB}$, cosine roll-off coefficient $=0.8$ )

\begin{tabular}{ccccccccccc}
\hline & ASK & FSK & MSK & BPSK & QPSK & OQPSK & 16QAM & AM & FM & Other \\
\hline (a) Method in this paper & $100 \%$ & $100 \%$ & $100 \%$ & $99 \%$ & $98 \%$ & $93 \%$ & $91 \%$ & $99 \%$ & $99 \%$ & $96 \%$ \\
(b) Azzouz's Method & $93 \%$ & $81 \%$ & - & $84 \%$ & $79 \%$ & - & - & $85 \%$ & $81 \%$ & - \\
(c) Method in this paper & $100 \%$ & $100 \%$ & $100 \%$ & $99 \%$ & $98 \%$ & $94 \%$ & $92 \%$ & $99 \%$ & $100 \%$ & $96 \%$ \\
(d) Azzouz's Method & $98 \%$ & $99 \%$ & - & $90 \%$ & $88 \%$ & - & - & $87 \%$ & $85 \%$ & - \\
\hline
\end{tabular}

Signals of $1 \mathrm{MHz}$ bandwidth under 8dB SNR, 1000 times per modulation mode. (a)(b) with $0.5 \mathrm{MHz}$ residual frequency offset and (c)(d) without that. The cosine roll-off coefficient of BPSK, QPSK and 16QAM is 0.8.

\section{Conclusions}

In this paper, a novel algorithm for open-set modulation recognition of communication signals is proposed. Firstly, the algorithms structure including pre-processing section and a two-stage modulation recognition section is developed. Besides, characteristic values, particularly two novel characteristics namely continuous coefficient of unification instant phase and concentration ratio of instant amplitude unification, are used for modulation modes including AM, FM, ASK, FSK, MSK, BPSK, QPSK, OQPSK, and 16QAM. Meanwhile, the best decision threshold value of each characteristic is determined through the simulation experiments. Finally, the recognition accuracy of nine modulation modes and others is presented under different signal-to-noise ratios. Computer simulation results demonstrate that the proposed recognition algorithms identify more species and reach the recognition accuracy better than $93 \%$ in $8 \mathrm{~dB}$ SNR value. In addition, it is noted that the algorithm is rarely sensitive to residual frequency offsets and available for MF signals. Meanwhile the cumulants are improved to reach a better robustness of square-root raised cosine roll-off coefficient.

\section{Acknowledgements}

The research work was supported by National Natural Science Foundation of China under Grant No. 61321061, 61132002, 61032003, Natural Basic Research Program of China under Grant No. 2013CB329000 and Tsinghua University Initiative Scientific Research Program under Grant No. $2011 \mathrm{Z} 05112$.

\section{References}

[1] Mitola Joe. The software radio architecture. Communications Magazine, IEEE, 33, Issue: 5, 26-38, 1995

[2] J.Lopatka, M.Pedzisz. Automatic modulation Classification using statistical moments and fuzzy classifier. Proc. IEEE ICSP，2000，3： 1500-1506

[3] Alyaoui N, Ben Hnia H, Kachouri A, et al. The modulation recognition approaches for software radio[C]//Signals, Circuits and Systems, 2008. SCS 2008. 2nd International Conference on. IEEE, 2008: 1-5. 
[4] O.A.Dobre, A.Abdi, Y.Bar-Ness, W.Su. Cyclostationarity-Based Blind Classification of Analog and Digital Modulations. Proc. MILCOM, 2006, 1-7

[5] Azzouz E. Automatic Modulation Recognition of Commumietion Signals[J]. Kluwer Academic Publishers, 1: 996.

[6] Yu B M, Hu G B. Intrapulse Modulation Recognition of Radar Signals Based on Ordered Time-Frequency Curve[J]. Telecommunication Engineering, 2012, 7: 010.

[7] Avci D. An intelligent system using adaptive wavelet entropy for automatic analog modulation identification[J]. Digital Signal Processing, 2010, 20(4): 1196-1206.

[8] Wang G H, Shen Y. A Novel Modulation Recognition Algorithms for Wireless Analog Signal[M]//Informatics and Management Science IV. Springer London, 2013: 109-118.

[9] Avci E, Hanbay D, Varol A. An expert discrete wavelet adaptive network based fuzzy inference system for digital modulation recognition[J]. Expert Systems with Applications, 2007, 33(3): 582-589.

[10] Hassan K, Dayoub I, Hamouda W, et al. Automatic modulation recognition using wavelet transform and neural networks in wireless systems[J]. EURASIP Journal on Advances in Signal Processing, 2010, 2010. 